\title{
Abdominal Ascariasis: Where to Focus on Imaging Studies
}

\author{
Jan Suhail ${ }^{1}$, Omair Shah ${ }^{2}$, Obaid Shah ${ }^{1}$, Jan Sarfaraz ${ }^{3}$, Irshad Mohuiddin ${ }^{1}$
}

\begin{abstract}
Objective: To assess the various presentations of abdominal ascariasis and their imaging features for developing a comprehensive radiological evaluation protocol.

Methods: Demographic and clinical profile of 84 patients with abdominal ascariasis was noted. Patients were divided into 2 groups with age less than 20 comprising Group A and those with age greater than 20 Group B. All the patients had a routine lab workup and a dedicated USG scan to look for objective evidence of intestinal or hepatobiliary ascariasis. Any history of previous intervention (cholecystectomy or ERCP) was also sought.

Results: Abdominal ascariasis is more common in females observed in $62 \%$ of our patients. Peripheral eosinophilia was observed in $70(83 \%)$ patients. We had $74(88 \%)$ patients with intestinal ascariasis with 2 patients having appendicular ascariasis. Biliary ascariasis was observed in $20(24 \%)$ patients and pancreatic ductal ascariasis in $6(7 \%)$ patients. We found peritoneal ascariasis in $1(2 \%)$ pediatric patient and $2(3 \%)$ patients in our study had ascariasis associated with the liver abscess. $\mathrm{OCH}$ was observed in 4 patients while 2 patients presented with worm cholecystitis.

Conclusion: Peripheral eosinophilia can be used to select patients in endemic regions for undergoing a dedicated USG scan. USG is the investigation of choice for both intestinal and hepatobiliary ascariasis. Worm migration is more common in adults and is especially prevalent in individuals with previous history of ERCP or cholecystectomy.
\end{abstract}

\section{Keywords}

USG Ultrasonography; ERCP Endoscopic Retrograde Cholangiopancreatography; ALP Alkaline Phosphatase; CBD Common Bile Duct

${ }^{1}$ Government Medical College, Srinagar, J\&K, India

${ }^{2}$ Sheri Kashmir Institute of Medical Sciences, SOURA, J\&K, India

${ }^{3}$ Directorate of Health Services, J\&K, India

${ }^{*}$ Corresponding author: shahomair133@gmail.com

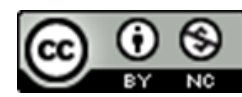

Copyright @Jan Suhail, Omair Shah, Obaid Shah, Jan Sarfaraz, Irshad Mohuiddin, 2020

\section{Introduction}

Ascariasis is the name given to the disease spectrum associated with infestation of humans by a nematode Ascariasis lumbricoides [1]. It is a common pathogen and affects approximately $33 \%$ of the world population [2]. This disease has been found to have a predilection for tropical and developing countries owing to the poor hygiene, malnutrition and poor quality of drinking water. The organs of involvement in humans include bowel (most common), hepatobiliary, pancreas and lungs. The presenting complaint depends on the organs of involvement, however in majority of the cases it is asymptomatic or presents only with vague abdominal pain. In pediatric age group, however it may be represented with growth stunting and essential nutrient deficiencies [3, 4]. Ascariasis presenting clinically however requires a heavy worm load described variably as between 13- 40 worms. In endemic areas, ascariasis is a frequent diagnosis and 
should be kept in mind as a cause of acute upper abdominal pain [5].

The infestation occurs through oral route with ingestion of the eggs and the adult worm lives in the small intestine. However, it has the tendency to enter other hollow cavities and can present in different ways including pneumonia, pancreatitis, cholangitis, intestinal obstruction \& perforation, appendicitis as well as liver abscess and hepatolithiasis. Oriental cholangiohepatitis is one chronic complication of biliary ascariasis that can be distressing with multiple and repeated hepatic calculi formation [6].

Ultrasonographic imaging remains the main stay of diagnosis as far as abdominal ascariasis is concerned. The worm is seen as long tubes with echogenic walls and a central anechoic canal representing the alimentary tract when viewed longitudinally and has round target like appearance on cross section [7]. In some cases, they may form a cluster within the gut or the biliary tract giving rise to spaghetti sign while dead worms may present as linear echogenic foci within the biliary tract.

Our study was aimed at exploring the various presentations of abdominal ascariasis and their imaging features so that a comprehensive radiological evaluation is devised in patients in our part of the world where the disease is quite common.

\section{Methods}

This study was an observational study conducted over a period of one year in our tertiary care center, Government Medical College (GMC) Srinagar, J\&K from March 2019 to April 2019. The patients in our study were referred to our department as cases of upper abdominal pain, jaundice, stunted growth and intestinal obstruction. All the patients underwent an USG assessment and we included only those patients with a USG documented abdominal ascariasis in our study.

We included a total of 84 patients in our study with USG documented abdominal ascariasis. The demographic profile of all the patients and their clinical presentation was noted at the time of the scan. A note was also made of the laboratory investigations including CBC, LFT, amylase and a specific note of eosinophilia was made. An eosinophil count of $>500 / \mu \mathrm{l}$ was taken to be suggestive of peripheral eosinophilia. All the patients were subjected to a USG of the abdomen first in the emergency department followed by a dedicated ascariasis scan in our department. All the scans were done using LOGIC GE S8 USG machine in our department. Both high (7-12 Hz) and low frequency $(3-5 \mathrm{~Hz})$ probes were used to scan the gut and visceral organs. A specific search was made to look for worms at specific sites including gut (stomach, small \& large intestine and appendix), gall bladder, intrahepatic ducts, CBD, pancreatic duct and the peritoneal cavity during the specific ascariasis scan. Any double echogenic structure in these areas with a central anechoic canal representing the alimentary tract of the worm was taken to be objective evidence of ascariasis infestation. A note was made of the site of the worm and the associated complications like pancreatitis, intestinal obstruction or perforation, hepatic abscess, cholangitis, appendicitis and hepatolithiasis. All these complications were diagnosed using USG with the support of clinical and laboratory findings. The values were expressed as mean $\pm \mathrm{SD}$ and a $\mathrm{p}$ value of less than 0.05 was taken to be significant.

\section{Results}

\section{Patient profile}

We included 84 patients in our study with a mean age of $30 \pm 4$ years (range 3-70 years). We divided the patients into two groups - Group A with age less than 20 years including $25(30 \%)$ patients and Group B with age above 20 years including $59(70 \%)$ patients. Among the 84 patients $52(62 \%)$ were females while 32 (38\%) were males.

\section{Clinical Presentation}

The most common presentation was upper abdominal pain present in $65(77 \%)$ patients. Other features included nausea \& vomiting [40 (48\%) patients], fever [10 (12\%) patients], stunted growth [5 (6\%) patients], intestinal obstruction [8 (9\%) patients] and jaundice [8 (9\%) patients]. 


\section{Laboratory Investigations}

Peripheral eosinophilia was very common and was found in $70(83 \%)$ of our patients. Other lab values that were found to be increased were based on the presentation with serum amylase raised in patients with pancreatitis [6 (7\%) patients] and raised ALP in $20(24 \%)$ patients with hepatobiliary ascariasis.

\section{USG Findings}

We found intestinal ascariasis in $74(88 \%)$ of our patients. These included all $25(100 \%)$ patients in pediatric group A and $49(83 \%)$ patients in the adult group B. Among these 74 patients worm bolus formation was observed in $20(27 \%)$ patients with $5(25 \%)$ of them having dilated gut loops. Majority of these patients presented with small intestinal [50 (68\%) patients] and gastric ascariasis [20 (27\%) patients] while $2(2.7 \%)$ presented with appendicular ascariasis and $2(2.7 \%)$ others with large intestinal ascariasis. The 2 patients with appendicular ascariasis belonged to pediatric group A.

Biliary ascariasis (CBD, GB \& intrahepatic ducts) was seen $20(24 \%)$ patients in our study and among these $7(35 \%)$ patients had exclusive biliary ascariasis without intestinal ascariasis while $13(65 \%)$ others had intestinal ascariasis also. Among these 20 patients $16(80 \%)$ were adults in group B while only $4(20 \%)$ patients belonged to pediatric group B. We also found $4(5 \%)$ patients in our study with imaging features of $\mathrm{OCH}$ and among these 2 had biliary ascariasis at the time of scan, 1 had presence of dead worm while 1 other had a history of biliary ascariasis with endoscopic removal. Also 2 (8\%) patients had GB ascariasis and presented with features of acute cholecystitis.

We found pancreatic ductal ascariasis in $6(7 \%)$ patients all of whom belonged to the adult group B. Among these $3(50 \%)$ patients had associated intestinal ascariasis while $3(50 \%)$ others had exclusive pancreatic ductal ascariasis.

We found peritoneal ascariasis in $1(2 \%)$ pediatric patient who had intestinal perforation and shock. $2(3 \%)$ patients in our study had ascariasis associated liver abscess.

\section{Previous Intervention}

We found that among 26 patients with hepatobiliary and pancreatic ascariasis $18(70 \%)$ patients had history of previous intervention in the form of cholecystectomy or ERCP with or without sphincterotomy. This is especially true of the patients with a diagnosis of $\mathrm{OCH}$ with $8(80 \%)$ out of ten patients having a previous history of intervention.

\section{Discussion}

This study was conducted in a tertiary care center over a period of 1 year and included 84 patients with a mean age of $30 \pm 4$ years and a male to female ratio of 32 (38\%): 52 (62\%). The patients were divided into 2 groups- Group A patients with age $<20$ years including $25(30 \%)$ patients and Group B with age $>20$ years including $59(70 \%)$ patients. This shows that there is no age predilection for abdominal ascariasis, however a female predominance is observed. This is concordant with the findings of Khuroo et al. [8].

The most common presentation in our study was upper abdominal pain present in 65 (77\%) patients. Other features included nausea \& vomiting [40 (48\%) patients], fever [10 (12\%) patients], stunted growth [5 (6\%) patients], intestinal obstruction [8 (9\%) patients] and jaundice [8 (9\%) patients]. Most of these symptoms are attributed to intestinal ascariasis causing mechanical obstruction as well as parasitizing on essential nutrients. Patients can also have biliary colic in cases with CBD (Fig. 1) or GB (Fig. 2) ascariasis following migration of the worm via the papilla. Biliary ascariasis can further lead to strictures, calculi, cholecystitis and pancreatitis. A few instances of worm associated liver abscess (Fig. 3) have also been described. In our study we had 20 (24\%) patients with biliary ascariasis with 7 having biliary ascariasis alone without evidence of intestinal ascariasis. Most of the patients (16) with biliary ascariasis belonged to the adult Group B. We also had 6 patients with pancreatic ductal ascariasis presenting with pancreatitis all of whom belonged to adult Group B. Thus it can be concluded that abdominal ascariasis can have a spectrum of presentations and migration of 
the worm via the papilla occurs more commonly in adults than in pediatric age group. A thorough search for the worms should be done in patients belonging to endemic area like ours with symptoms suggestive of parasitic infestation. These findings are concordant with those of Mani S et al. [9] and Louw JH [10].

In our study we found that peripheral eosinophilia was present in a significant proportion of our patients. $70(84 \%)$ of our study population had this finding. Other lab values like ALP and serum amylase were found to be high in patients presenting with pancreatitis and biliary ascariasis. We can conclude that peripheral eosinophilia can be used as a sensitive marker to identify patients requiring a dedicated scan for abdominal ascariasis. This finding is consistent with the observations made by Pawlowski ZS [11], Sun T [12] and Gutierrez $\mathrm{Y}$ [13] in their studies.

We found intestinal ascariasis in $74(88 \%)$ of our patients. These included all the 25 (100\%) patients in pediatric group A and 49 (83\%) patients in the adult group B. Among these 74 patients worm bolus formation (Fig. 4) was seen in $20(27 \%)$ patients with $5(25 \%)$ of them having dilated gut loops. Majority of these patients presented with small intestinal [50 (68\%) patients] and gastric ascariasis (Fig. 5) [20 (27\%) patients] while $2(2.7 \%)$ presented with appendicular ascariasis (Fig. 6) and 2 (2.7\%) others with large intestinal ascariasis. The 2 patients with appendicular ascariasis belonged to pediatric group A. The patients with gastric ascariasis had associated worm bolus formation in the distal bowel suggesting some form of obstruction leading the worms to move retrograde into stomach. Thus in pediatric age group ascariasis is predominantly intestinal and appendicitis as a complication is more common in this age group. Thus pediatric patients presenting with features of appendicitis in endemic areas, a possibility of worm appendicitis should be kept in mind.

Biliary ascariasis (CBD, GB \& intrahepatic ducts) was seen in $20(24 \%)$ patients in our study and among these 7 (35\%) patients had exclusive biliary ascariasis without intestinal ascariasis while $13(65 \%)$ others had intestinal ascariasis also. Among these 20 patients $16(80 \%)$ were adults in group B while only $4(20 \%)$ patients belonged to pediatric group B. Also pancreatic ductal ascariasis (Fig. 7) was seen in $6(7 \%)$ patients all of whom belonged to the adult group B. Among these $3(50 \%)$ patients had associated intestinal ascariasis while $3(50 \%)$ others had exclusive pancreatic ductal ascariasis. This suggests that worm migration is more common in adults than in pediatric age group and a thorough search for any of these should be made while scanning patients with intestinal ascariasis to prevent long term complications associated with biliary ascariasis including strictures, calculi and oriental cholangiohepatitis. We also found peritoneal ascariasis (Fig. 8) in $1(2 \%)$ pediatric patient who had intestinal perforation and shock. $2(3 \%)$ patients in our study had ascariasis associated liver abscess. Also 2 (8\%) patients had GB ascariasis and presented with features of acute cholecystitis on USG in the form of GB wall thickening and pericholecystic fluid. Thus abdominal ascariasis should be taken as a spectrum far beyond intestinal ascariasis and its recognition and early treatment is essential to prevent long term devastating complications. Our findings are concordant with the observations of many previous studies including those of Khuroo et al. [8], Mani S et al. [9], Louw JH [10] and Pinus J [14].

We also found $4(17 \%)$ patients among those with biliary ascariasis in our study with imaging features of $\mathrm{OCH}$ and among these 2 had biliary ascariasis at the time of scan, 1 had presence of dead worm while 1 other had a history of biliary ascariasis with endoscopic removal. Thus we can say that $\mathrm{OCH}$ is an important and morbid complication of biliary ascariasis which is quite common in an endemic area like ours. Also OCH can even be an initial presentation of neglected biliary ascariasis and occurs more commonly in patients with previous history of intervention and biliary ascariasis. This group of patients would therefore require a regular and meticulous imaging follow up. These findings are in concordance with the studies conducted by Lim JH [15], Sperling RM [16] and Shah et al. [17].

In our study we found previous history of an intervention in the form of cholecystectomy or ERCP 


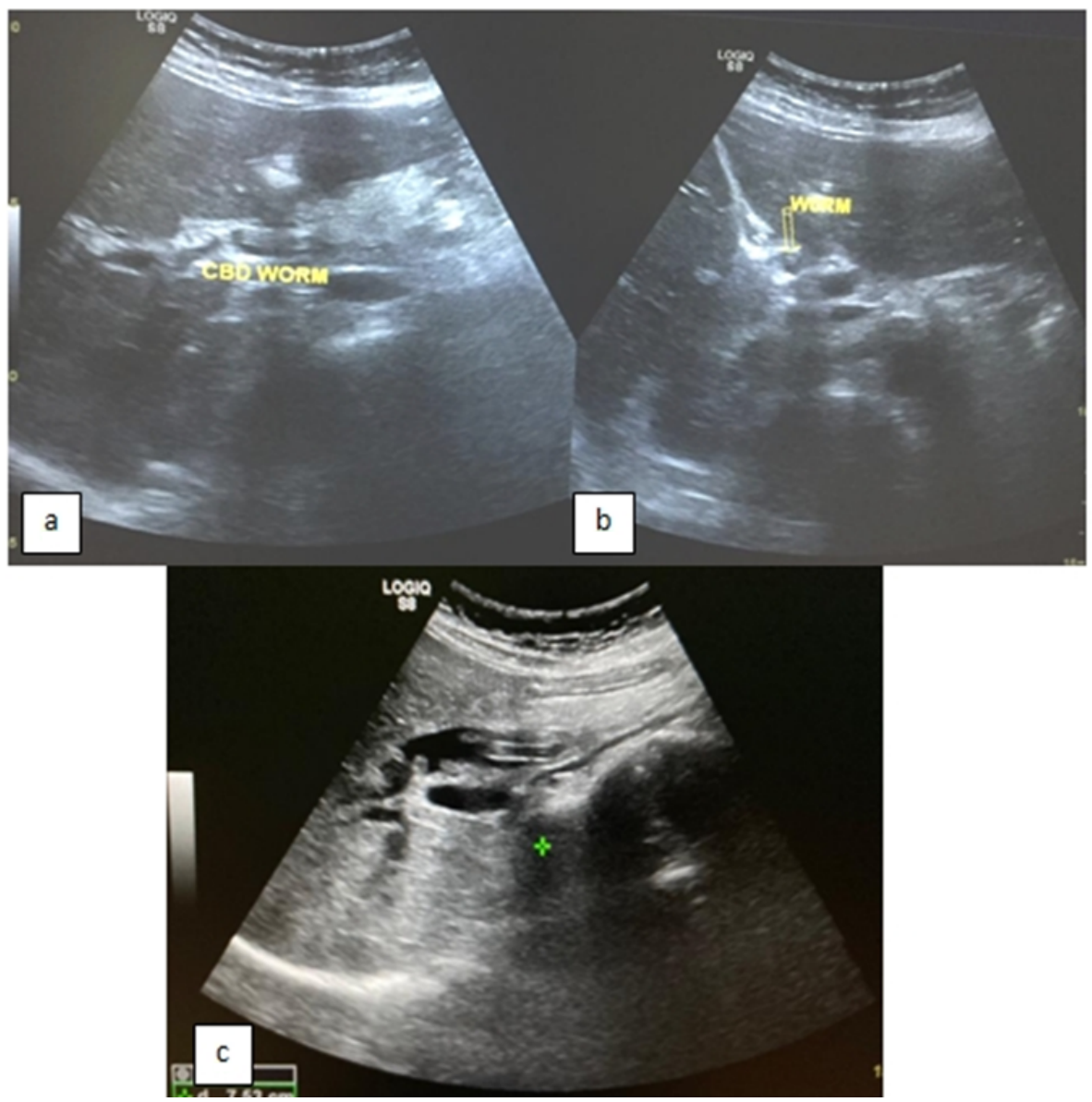

Figure 1. USG image showing CBD ascariasis in longitudinal and cross sectional view (a\& b). USG image in a different patient showing double linear echogenic worm within the CBD (c).

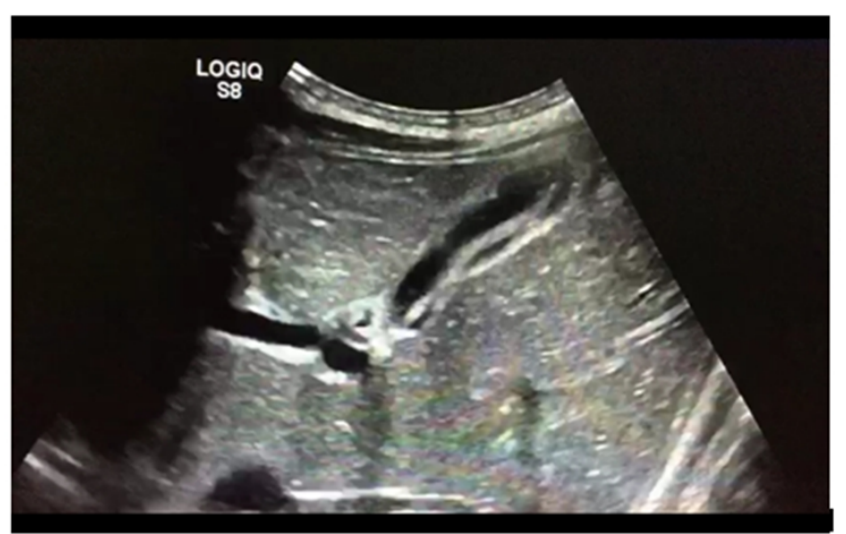

Figure 2. USG image of right upper quadrant clearly showing the worm within GB reaching up to the fundus.

in $18(70 \%)$ of the patients with biliary or pancreatic ascariasis. This suggests a predilection of worm

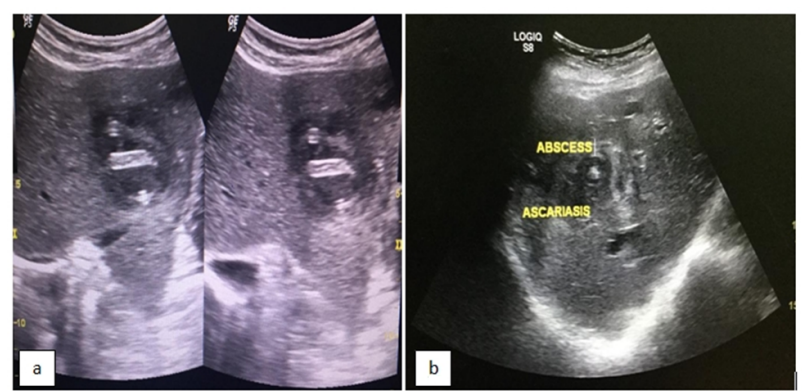

Figure 3. USG image showing double linear echogenic structure within a left lobe liver abscess

(a). USG image in a different patient showing worm associated right lobe abscess (b).

migration in these patients probably owing to the lax sphincter of Oddi in these patients. Thus patients with such history in endemic zones should undergo regular USG scans to look for hepatobil- 

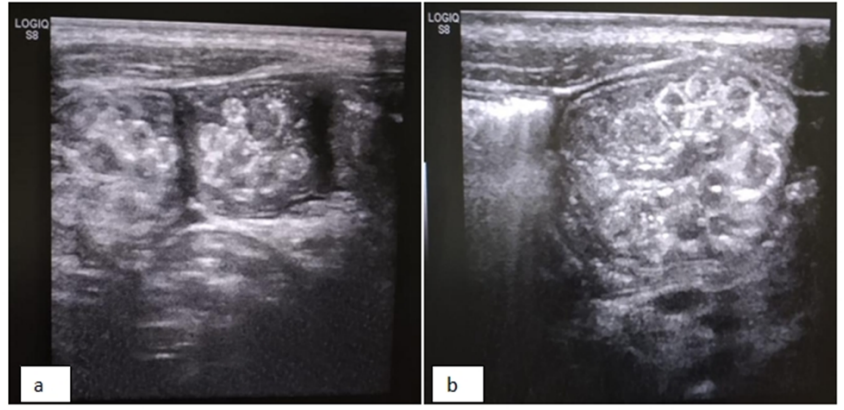

Figure 4. High frequency linear probe images showing two worm boluses (a) and a large worm bolus in another patient who also had dilated gut loops and presented with small bowel obstruction (b).

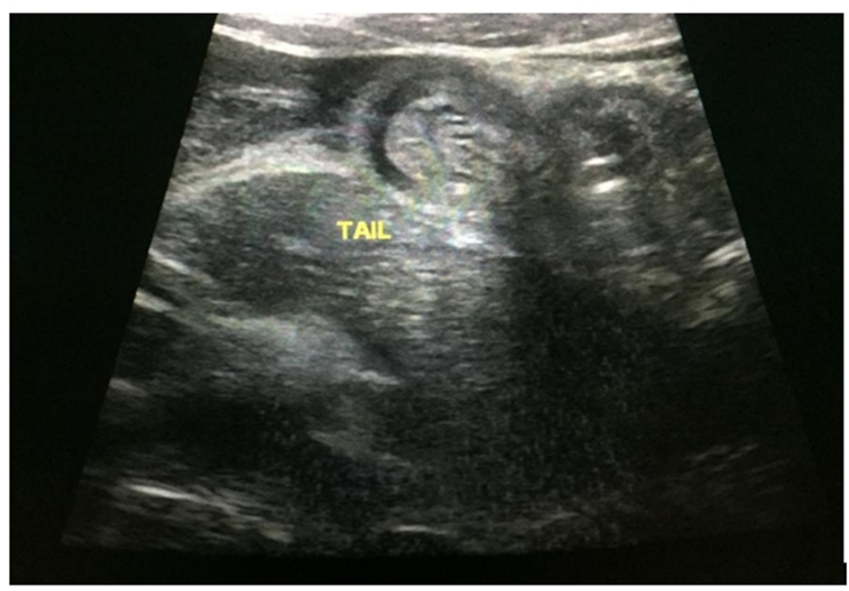

Figure 5. USG image of the epigastric region showing a bolus of worms in the stomach anterior to the tail of pancreas.

iary ascariasis and get promptly treated in order to prevent the morbidity associated with worm complications. These observations were also made by Khuroo MS et al. [8], Sandouk F [18] et al. and Mukhopadhyay M [19] in their studies.

The limitations of our study include a relatively small number of patients which could probably be because our center being a tertiary care center received only those patients that were symptomatic leaving a vast majority of the asymptomatic patients for primary health care facilities. Also our study did not follow up patients over a period of time to look for any long term complications and the efficacy of various treatments employed.

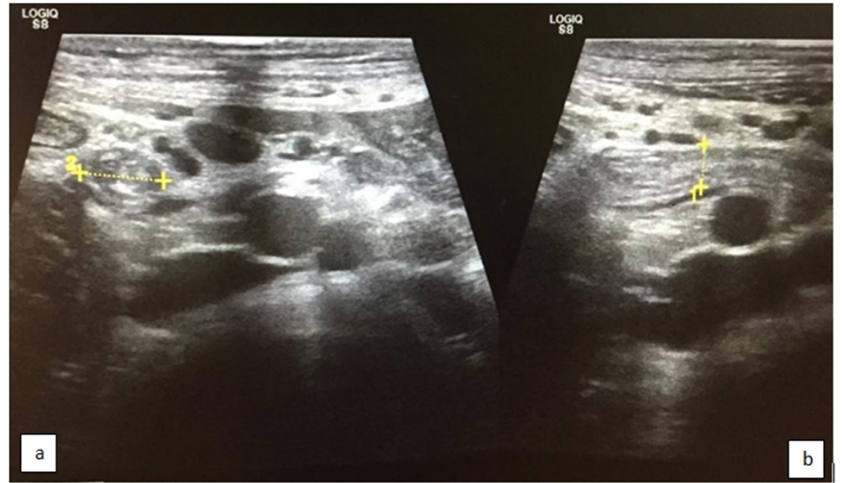

Figure 6. USG images cross sectional (a) and longitudinal (b) showing double linear echogenic structure within the appendix anterior to iliac vessels. The patient also had free fluid in pelvis and typical clinical features of appendicitis.

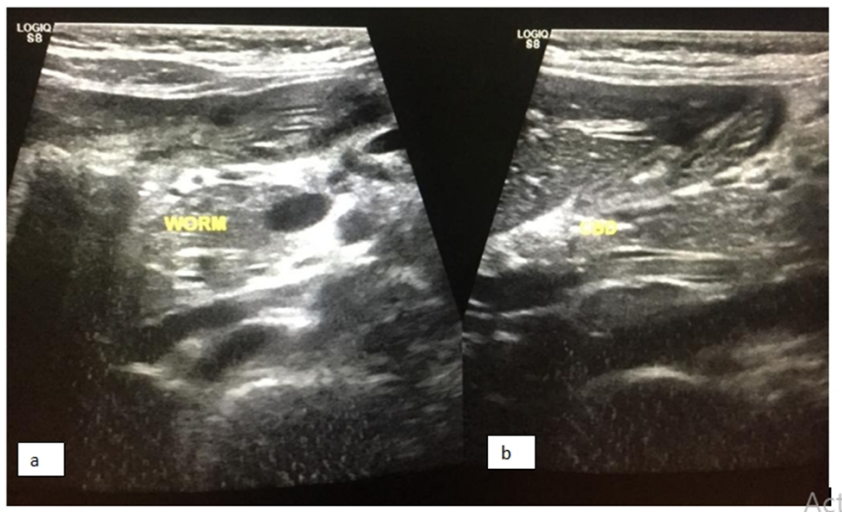

Figure 7. USG images showing pancreatic duct filled with worm in cross section (a) and longitudinal (b) views in the pancreatic head region.

\section{Conclusions}

Abdominal ascariasis has a varied presentation with involvement of many organ systems. In endemic area like ours, ascariasis should be in the list of causes for pancreatitis, appendicitis, hepatic abscess, intestinal obstruction/perforation, cholecystitis and intra hepatic calculi. USG is the investigation of choice for both intestinal and hepatobiliary ascariasis. Peripheral eosinophilia is one lab investigation that can be used to select patients in endemic regions for undergoing a dedicated USG scan. Worm migration is more common in adults and is especially prevalent in individuals with pre- 


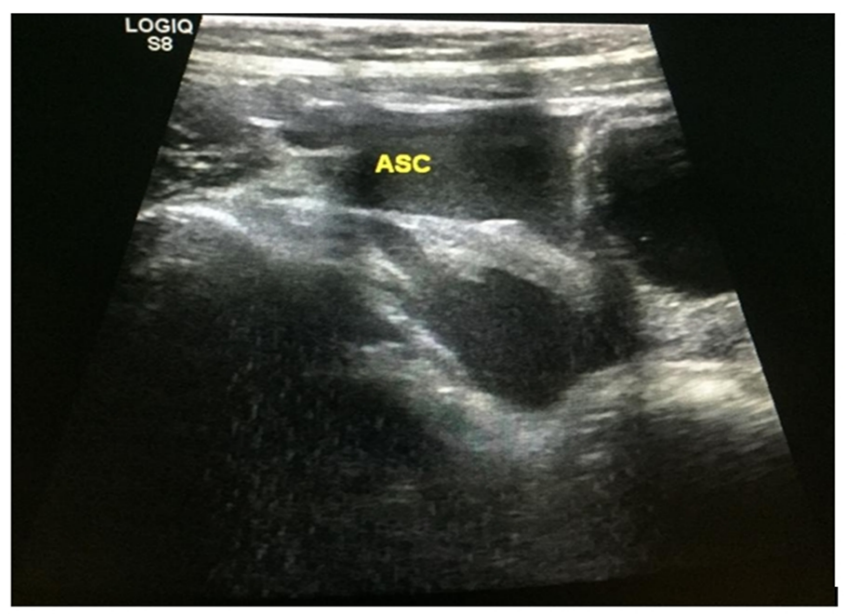

Figure 8. USG image of the pelvic area showing echogenic ascites with free floating worm in the peritoneal cavity in a patient with intestinal perforation.

vious history of ERCP or cholecystectomy.

\section{Informed Consent}

Written informed consent was obtained from the patient who participated in this case.

\section{Conflict of Interest}

The authors stated no conflict of interest.

\section{Financial Disclosure}

The authors declared no financial support.

\section{References}

[1] Gutierrez Y. Ascaridida - Ascaris, Lagochilascaris, Anisakis, Pseudoterranova and Baylisascaris. In: Gutierrez Y, editor. Diagnostic Pathology of Parasitic Infections with Clinical Correlations. Philadelphia: Lea \& Febiger; 1990. p. 236-247.

[2] Bundy DA, Cooper ES, Thompson DE, Anderson RM, Didier JM. Age-related prevalence and intensity of Trichuris trichiura infection in a St. Lucian community. Trans
R Soc Trop Med Hyg. 1987; 81: 8594. DOI: https://doi.org/10.1016/ 0035-9203(87) 90293-8

[3] Stephenson LS, Latham MC, Kinoti SN, Kurz $\mathrm{KM}$, Brigham H. Improvements in physical fitness of Kenyan schoolboys infected with hookworm, Trichuris trichiura and Ascaris lumbricoides following a single dose of albendazole. Trans R Soc Trop Med Hyg. 1990; 84: $277-$ 282. DOI: https://doi.org/10.1016/ 0035-9203(90) 90286-N

[4] Nokes C, Bundy DA. Does helminth infection affect mental processing and educational achievement? Parasitol Today. 1994; 10: 1418. DOI: https://doi.org/10.1016/ 0169-4758(94) 90348-4

[5] de Silva NR, Chan MS, Bundy DA. Morbidity and mortality due to ascariasis: Re-estimation and sensitivity analysis of global numbers at risk. Trop Med Int Health. 1997; 2: 519-528. DOI: https://doi.org/10.1046/ j.1365-3156.1997.d01-320.x [PMid:9236818]

[6] Langewar DN, Maheshwari MB, Wegholikar UL. Hepatic perforation due to ascariasis. Indian J Pediatr. 1993; 60: $457-$ 459. DOI: https://doi.org/10.1007/ BF 02751215 [PMid:8253499]

[7] Hoffmann H, Kawooya M, Esterre P, Ravaoalimalala VE, Roth J, Thomas AK, et al. In vivo and in vitro studies on the sonographical detection of Ascaris lumbricoides. Pediatr Radiol [Internet]. 1997 Mar 19;27(3):226-9. DOI: https://doi.org/10.1007/ s 002470050106 [PMid:9126575]

[8] Khuroo MS, Zargar SA, Mahajan R. Hepatobiliary and pancreatic ascariasis in India. Lancet. 1990; 335: 1503-1506. DOI: https://doi.org/10.1016/ 0140-6736(90) 93037-P

[9] Mani S, Merchant H, Sachdev R, Rananavare $\mathrm{R}$, Cunha N. Sonographic evaluation of biliary 
ascariasis. Australas Radiol. 1997; 41: 204206. DOI: https: / / doi .org/10.1111/ j.1440-1673.1997.tb00718.x [PMid:9153828]

[10] Louw JH. Biliaray ascariasis in childhood. S Afr J Surg 1974; 12: 19-25.

[11] Pawlowski ZS. Ascariasis. In: Warran KS, Mahmoud AA, editors. Tropical and Geographical Medicine. 2nd ed. New York: McGrawHill; 1990. p. 369.

[12] Sun T. Ascariasis. In: Sun T, editor. Pathology and Clinical Features of Parasitic Diseases. New York: Masson; 1980. p. 115-120.

[13] Gutierrez Y. Ascaridida - Ascaris, Lagochilascaris, Anisakis, Pseudoterranova and Baylisascaris. In: Gutierrez Y, editor. Diagnostic Pathology of Parasitic Infections with Clinical Correlations. Philadelphia: Lea \& Febiger; 1990. p. 236-247.

[14] Pinus J. Surgical complications of ascariasis. Prog Pediatr Surg 1982; 15: 79-86.

[15] Lim JH. Oriental cholangiohepatitis: Pathologic, clinical, and radiologic features. AJR Am J Roentgenol. 1991; 157: 1-8. DOI: https://doi.org/10.2214/ajr. 157.1.2048504 [PMid:2048504]

[16] Sperling RM, Koch J, Sandhu JS, Cello JP. Recurrent pyogenic cholangitis in Asian immigrants to the United States: Natural history and role of therapeutic ERCP. Dig Dis Sci. 1997; 42: $865-871$.

[17] Shah OJ, Zargar SA, Robbani I. Biliary Ascariasis: A Review. World J Surg [Internet]. 2006 Aug 10;30(8):1500-6. DOI: https: / / doi . org/10.1007/s00268-005-0309-1

[PMid:16874446]

[18] Sandouk F, Haffar S, Zada MM, Graham DY, Anand BS. Pancreaticbiliary ascariasis: Experience of 300 cases. Am J Gastroenterol. 1997; 92: 2264-2267.
[19] Mukhopadhyay M. Biliary ascariasis in the Indian subcontinent: A study of 42 cases. Saudi J Gastroenterol [Internet]. 2009 Aug 10;15(2):121. DOI: https://doi. org/10.4103/1319-3767.48970

[PMid:19568577 PMCid:PMC2702965]

Received: $2020-07-27$

Revised: $2020-08-15$

Accepted: 2020-08-19 